\title{
A CRITERION FOR WEAK CONVERGENCE OF MEASURES WITH AN APPLICATION TO CONVERGENCE OF MEASURES ON $D[0,1]$
}

\author{
FLEMMING TOPSØE
}

\section{Introduction.}

The usual way to establish weak convergence of a sequence of probability measures on $C[0,1]$ is to prove that all the finite dimensional distributions converge weakly to the "right" limits and that the sequence is tight; then a theorem, which is quite easy to establish, tells us that we have weak convergence. If we turn our attention to the Skorohod space $D[0,1]$ we find that the analogous theorem is much harder to obtain. Recently, P. Billingsley has obtained a suitable result published in [1, p. 124]. My aim has been to find a general theorem, valid in any Polish space, which implies the desired result in $D[0,1]$.

All measures below are supposed to be defined on the Borel $\sigma$-field.

A reasonable problem inspired by the concrete question about $D[0,1]$ is the following: Let $X$ be a Polish space (that is, separable and metrizable in such a way that it becomes complete), and let $\mathscr{A}$ be a field of Borel sets generating the entire Borel $\sigma$-field $\mathscr{B}(X)$. If $P,\left(P_{n}\right)_{n \geqq 1}$ are probability measures on $X$ such that $\left(P_{n}\right)$ is tight and such that $P_{n} A \rightarrow P A$ for all sets $A$ in $\mathscr{A}$, is it then true, or under what additional assumptions is it true, that $P_{n}$ converges weakly to $P$ ?

Equivalently, we could ask if, under the just mentioned hypotheses on $X$ and $\mathscr{A}$, the facts that $P_{n} \rightarrow Q$ for some probability measure $Q$ and $P_{n} A \rightarrow P A$ for all $A$ in $\mathscr{A}$, imply $P=Q$. Here, as below, the sign $\rightarrow$ is used to indicate weak convergence of probability measures as well as ordinary convergence of real numbers.

Since $P_{n} \rightarrow Q$ and $P_{n} A \rightarrow P A$ imply

$$
Q \stackrel{\circ}{A} \leqq \liminf P_{n} \stackrel{\circ}{A} \leqq P A \leqq \limsup P_{n} \bar{A} \leqq Q \bar{A}
$$

a problem related to the problem above arises: Let again $X$ be Polish and $\mathscr{A}$ a field generating $\mathscr{B}(X)$. If $P$ and $Q$ are two probability measures

Received November 13, 1967; in revised form January 28, 1969.

Math. Scand. $25-7$ 


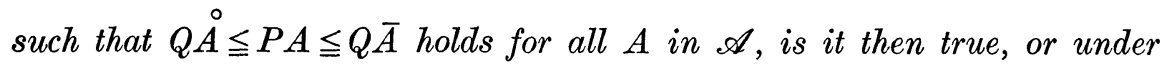
what additional assumptions is it true, that $P=Q$ ?

Without additional assumptions the answers to both problems are negative. Indeed, $\mathrm{O}$. Björnsson observed that there is a counterexample in which $P$ and $Q$ are as simple as possible, namely point masses.

\section{A convergence criterion.}

A class $\mathscr{A}$ of subsets of the topological space $X$ is said to separate points $T_{2}$ if, for every pair of distinct points $x$ and $y$ in $X$ there exists a set $A$ in $\mathscr{A}$ such that $x \in \AA$ and $y \notin \bar{A}$.

Theorem 1. Let $\mathscr{A}$ be a lattice of subsets of the Polish space $X$ and suppose that $\mathscr{A}$ separates points $T_{\mathbf{2}}$.

(i) If $P$ and $Q$ are probability measures on $X$ such that $Q \stackrel{\circ}{P} \overline{\bar{A}}$ for all $A \in \mathscr{A}$, then $P=Q$.

(ii) If $P,\left(P_{n}\right)_{n \geqq 1}$ are probability measures on $X$ such that $\left(P_{n}\right)_{n \geqq 1}$ is tight and such that $\lim \sup P_{n} A \leqq P \bar{A}$ for all $A \in \mathscr{A}$, then $P_{n} \rightarrow P$.

Part (ii) is the hoped for convergence criterion.

Proof. (i) If $K_{1}$ and $K_{2}$ are disjoint compact subsets of $X$, we can find $A \in \mathscr{A}$ such that $K_{1} \subset A$ and $\bar{A} \cap K_{2}=\varnothing$. It follows that $Q K_{1} \leqq 1-P K_{2}$. Employing the tightness of $P$ and $Q$, one deduces from this that $P=Q$.

(ii) Let $\left(P_{n_{k}}\right)_{k \geqq 1}$ be a convergent subsequence of $\left(P_{n}\right)_{n \geqq 1}$, say $P_{n_{k}} \rightarrow Q$. Then

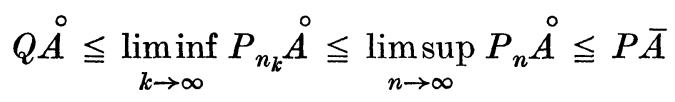

for all $A \in \mathscr{A}$. By (i), $Q=P$ follows.

Part (i) in the theorem can of course be considered as a special case of part (ii). It is easy to see, by means of a simple counterexample, that we can not drop the assumption that $\mathscr{A}$ be a lattice. We have been unable to decide whether one can relax this assumption assuming only that $\mathscr{A}$ is closed under finite intersections. The condition in Theorem 1 that $\mathscr{A}$ separates points $T_{2}$ is necessary, as is easily seen.

It is not difficult to extend the convergence criterion from measures on Polish spaces to tight measures (Radon measures) on arbitrary Hausdorff spaces; also, one may consider a lattice of functions in stead of a lattice of sets. It is our intention to publish elsewhere some results on weak convergence of tight measures on arbitrary Hausdorff spaces. 
If $\mathscr{A}$ in the convergence criterion is a subclass of $\mathscr{B}(X)$, then $\mathscr{A}$ generates $\mathscr{B}(X)$; to see this, note that $\mathscr{A}$ contains a countable subclass separating points and apply Theorem 3.3 of [3]. This argument was pointed out to us by E. T. Kehlet.

\section{An application to measures on the Skorohod space $D[0,1]$.}

$D[0,1]$ consists of those real-valued functions on $[0,1]$ which are continuous from the right for $0 \leqq t<1$ and have limits from the left for $0<t \leqq 1$. The distance $d(x, y)$ between two functions in $D[0,1]$ is the infimum of those $\varepsilon \geqq 0$ for which there exists an increasing homeomorphism $\lambda$ from $[0,1]$ onto itself such that $\|\lambda-i\|<\varepsilon$ and $\|x-y \circ \lambda\|<\varepsilon$; here $i$ denotes the identity map and $\|\cdot\|$ the uniform norm. The metric space $D[0,1]$ is known to be Polish. For a finite (ordered) subset $t=$ $\left\{t_{1}, \ldots, t_{k}\right\}$ of $[0,1]$ we denote by $\pi_{\boldsymbol{t}}$ the projection from $D[0,1]$ onto $\mathrm{R}^{k}$. The projections are all measurable. For a probability measure $P$ on $D[0,1]$ we denote by $T_{P}$ the set of $t \in[0,1]$ such that $\pi_{t}$ is continuous a.e. $P$. There are at most countably many points in $[0,1] \backslash T_{P}$; these points are called fixed points of discontinuity for $P$.

The purpose of this section is to prove the following result:

THEOREM 2. Let $\left(P_{n}\right)_{n \geqq 1}$ be a tight sequence of probability measures on $D[0,1]$ and let $T$ be a dense subset of $[0,1]$ containing the point 1 . Suppose that for each finite subset $\boldsymbol{t}$ of $T$ there is a probability measure $P_{\boldsymbol{t}}$ on the proper Euclidean space such that $P_{n} \pi_{t}^{-1}$ converges weakly to $P_{t}$. Then the sequence $\left(P_{n}\right)_{n \geqq 1}$ converges weakly. Furthermore, the limit measure $P$ can be identified by the formula

$$
P \pi_{t}{ }^{-1}=P_{t+},
$$

which holds for any finite subset $\boldsymbol{t}$ of $[0,1]$.

The formula $P \pi_{\boldsymbol{t}}{ }^{-1}=P_{\boldsymbol{t}+}$ means, first of all, that the limit from the right, in the sense of weak convergence, exists at $t$ and, secondly, that this limit is the finite dimensional distribution of $P$ at $t$. In more detail, what we claim is the following: Let $\boldsymbol{t}=\left\{t_{1}, \ldots, t_{k}\right\}$ be any finite subset of $[0,1]$; let, for each $v=1,2, \ldots$,

$$
\boldsymbol{t}_{v}=\left\{t_{\nu 1}, \ldots, t_{v k}\right\}
$$

be a finite subset of $T$ with $t_{v i}>t_{i}$ for each $i=1, \ldots, k$ (unless $t_{i}=1$ in which case we demand $t_{v i}=1$ ); suppose further that

$$
t_{v i} \downarrow t_{i} \quad \text { as } \nu \rightarrow \infty \quad \text { for each } i=1, \ldots, k
$$


(shortly: $\boldsymbol{t}_{v} \downarrow \boldsymbol{t}$ ). Then we claim that the measures $P_{\boldsymbol{t}_{v}}$ converge weakly in $\mathrm{R}^{k}$ to $P \pi_{\boldsymbol{t}}{ }^{-1}$ as $\nu \rightarrow \infty$.

In Theorem 2, the limit measure is not supposed to be known in advance; thus the result can be used to construct various measures.

A special case of Theorem 2 (with known limit measure and special $T$ ) has been established by Billingsley (Theorem 15.1 of [1]). Our method is completely different from Billingsley's.

To prove that $\left(P_{n}\right)$ converges weakly in Theorem 2 we need some simple lemmas.

Lemma 1. For any finite subset $\boldsymbol{t}=\left\{t_{1}, \ldots, t_{k}\right\}$ of $[0,1]$ and any subset $E$ of $\mathrm{R}^{k}$ we have

$$
\pi_{t}^{-1}(\bar{E}) \subset \overline{\pi_{t}^{-1}(E)} \quad \text { and } \quad \pi_{t}^{-1}(\stackrel{\circ}{E}) \supset \widetilde{\circ} \stackrel{\circ}{\pi_{t}^{-1}(E)} .
$$

In other words, all projections are open mappings.

Proof. Assume that $x \in \pi_{\boldsymbol{t}}^{-1}(\bar{E})$. Then $\left(x\left(t_{1}\right), \ldots, x\left(t_{k}\right)\right)$ lies in $\bar{E}$. Thus, to any $\delta>0$ we can find real numbers $r_{1}, \ldots, r_{k}$ with $\left|r_{i}\right|<\delta$ for all $i$, and such that $\left(x\left(t_{1}\right)+r_{1}, \ldots, x\left(t_{k}\right)+r_{k}\right)$ lies in $E$. Clearly then, there exists a function $y$ in $D$ with $y\left(t_{i}\right)=x\left(t_{i}\right)+r_{i}$ for all $i$, and such that the uniform distance from $x$ to $y$ is less than $\delta$. Then the Skorohod distance from $x$ to $y$ is also less than $\delta$. Since $y \in \pi_{t}^{-1}(E)$ and $\delta$ is arbitrary the first inclusion follows. The second inclusion is a consequence of the first.

Lemma 2. Let $s$ be a point in $[0,1]$ and $E$ a subset of R. Denote by $A$ the cylinder set $\pi_{s}{ }^{-1}(E)=\{x \in D: x(s) \in E\}$. If $0<s<1$, then we have

$$
\begin{aligned}
\bar{A} & =\{x \in D: x(s-) \in \bar{E} \text { or } x(s) \in \bar{E}\}, \\
\stackrel{\circ}{A} & =\{x \in D: x(s-) \in \stackrel{\circ}{E} \text { and } x(s) \in \stackrel{\circ}{E}\} .
\end{aligned}
$$

If $s$ is either 0 or 1 , then

$$
\begin{gathered}
\bar{A}=\{x \in D: x(s) \in \bar{E}\}, \\
\stackrel{\circ}{A}=\{x \in D: x(s) \in \stackrel{\circ}{E}\} .
\end{gathered}
$$

Proof. The case $s=0$ or 1 is easily treated. Now assume that $s \in(0,1)$. If $x \in \bar{A}$ then there exists a sequence $\left(x_{n}\right)$ of functions in $A$ and a sequence $\left(\lambda_{n}\right)$ of increasing homeomorphisms of $[0,1]$ onto $[0,1]$ such that

$$
\left\|\lambda_{n}-i\right\| \rightarrow 0 \text { and }\left\|x_{n}-x \circ \lambda_{n}\right\| \rightarrow 0 \text {. }
$$


Put $s_{n}=\lambda_{n}(s)$. We may assume that either $s_{n}<s$ holds for all $n$ or else $s_{n} \geqq s$ holds for all $n$. If the first alternative takes place, then $x\left(s_{n}\right) \rightarrow x(s-)$ and one finds that $x_{n}(s) \rightarrow x(s-)$ so that $x(s-) \in \bar{E}$. The second alternative leeds to $x(s) \in \bar{E}$.

To prove the reverse inclusion, assume first that $x(s) \in \bar{E}$; it follows from Lemma 2 that $x \in \bar{A}$. Now assume that $x(s-) \in \bar{E}$. By moving the function a little to the right and then adding a small constant function, one arrives at a function in $A$. Intuitively, it is thus clear that $x \in \bar{A}$. It is left to the reader to make this argument rigorous.

LemMa 3. Let $T$ be a dense subset of $[0,1]$ containing the point 1 . Denote by $\mathscr{A}$ the class of cylinder sets based on time-points in $T$, that is, $\mathscr{A}$ is the class of all sets $\pi_{\boldsymbol{t}}^{-1}(E)$, where $\boldsymbol{t}$ ranges over all finite subsets of $T$ and $E$ ranges over all Borel subsets of the proper Euclidean spaces. Then $\mathscr{A}$ is a field separating points $T_{\mathbf{2}}$.

Proof. Clearly, $\mathscr{A}$ is a field. We shall prove that $\mathscr{A}$ separates points $T_{2}$. Let $x$ and $y$ be distinct functions in $D$. This means that, for some $s$ in $[0,1], x(s)$ is distinct from $y(s)$. We shall assume that $x(s)<y(s)$ holds.

If $s=1$, then the set $A=\pi_{1}{ }^{-1}((-\infty, m))$, where $m$ is the midpoint of $[x(1), y(1)]$ lies in $\mathscr{A}$ and by Lemma 2 we also find that $x \in A$ and $y \notin \bar{A}$.

If $s<1$, we argue as follows. First choose three real numbers $m_{1}, m$ and $m_{2}$ such that $x(s)<m_{1}<m<m_{2}<y(s)$. Then, by the right continuity, we can find a positive $\delta$ with $s+\delta<1$ such that $x(t) \leqq m_{1}$ and $y(t) \geqq m_{2}$ hold for any $t$ in $(s, s+\delta)$. Since $T$ is dense in $[0,1]$, we can find a $t$ from $T$ in $(s, s+\delta)$. Now put $A=\pi_{t}^{-1}((-\infty, m)) . A$ lies in $\mathscr{A}$ and by Lemma 1 we also find that $x \in A$ and $y \notin \bar{A}$. Thus $\mathscr{A}$ separates points $T_{2}$.

Proof of the weak convergence in Theorem 2. We begin by remarking that the family of measures $\left(P_{t}\right)$ where $\boldsymbol{t}$ ranges over all finite subsets of $T$ is consistent. Now, let $Q_{1}$ be any limit measure for $\left(P_{n}\right)$, say $P_{n^{\prime}} \rightarrow Q_{1}$. Consider a finite subset $\boldsymbol{t}=\left\{t_{1}, \ldots, t_{k}\right\}$ of $T$ and a $k$-dimensional Borel set $E$. Then

$$
\begin{aligned}
& Q_{1}\left(\stackrel{\circ}{\pi_{t}^{-1} E}\right) \leqq \liminf P_{n^{\prime}}\left(\stackrel{\circ}{\pi_{t}^{-1} E}\right) \leqq \liminf P_{n^{\prime}}\left(\pi_{t}^{-1} \stackrel{\circ}{E}\right) \\
& \leqq \limsup P_{n^{\prime} \pi_{\boldsymbol{t}}^{-1}(\bar{E}) \leqq P_{\boldsymbol{t}}(\bar{E})},
\end{aligned}
$$

that is, we have

$$
Q_{1}\left(\frac{\circ}{\pi_{t}^{-1} E}\right) \leqq P_{t}(\bar{E})
$$


If $Q_{2}$ is another limit measure for $\left(P_{n}\right)$ then we find in an analogous manner

$$
P_{\boldsymbol{t}}\left(\stackrel{\circ}{E)} \leqq Q_{2}\left(\overline{\pi_{\boldsymbol{t}}^{-1} E}\right) .\right.
$$

Consider the class $\mathscr{A}$ of those subsets $A$ of $D[0,1]$ for which there exist a finite subset $\boldsymbol{t}=\left\{t_{1}, \ldots, t_{k}\right\}$ of $T$ and a $k$-dimensional Borel set $E$ such that $A=\pi_{\boldsymbol{t}}^{-1} E$ and $P_{t}(\partial E)=0$. Here $\partial E$ is the boundary in $\mathrm{R}^{k}$ of $E$. In checking which sets $A$ belong to $\mathscr{A}$ it does not matter which representation we use for $A$. Clearly, $\mathscr{A}$ is a field. The proof of Lemma 3 shows that $\mathscr{A}$ separates points $T_{2}$. By (2) and (3), the inequality $Q_{1}\left(\stackrel{\circ}{)} \leqq Q_{2}(\bar{A})\right.$ holds for any set $A$ in $\mathscr{A}$. By Theorem $1, Q_{1}$ and $Q_{2}$ are identical.

We shall now prove (1). In case the limit measure $P$ has only finitely many fixed points of discontinuity, this formula obviously holds. In the general case we use a rather elaborate argumentation and we begin with a lemma.

Lemma 4. Let $x$ be a function in $D[0,1]$ and $t$ a point in $[0,1]$ such that $|x(t)-x(t-)|<\varepsilon$. Then there exists a positive $\delta$ and a positive $h$ such that any element in the open sphere $S(x, \delta)$ with center $x$ and radius $\delta$ oscillates by less than $\varepsilon$ in the interval $[t-h, t+h]$.

This result is obvious.

Proof of (1) of Theorem 2. To ease the notation, we shall assume that the finite subset $t$ that we consider in fact only contains one point $t_{0}$. We may also assume that $t_{0}<1$. What we have given is a sequence $\left(s_{k}\right)$ of points in $T$ with $s_{k}>t_{0}$ for all $k$ and $s_{k} \downarrow t_{0}$ as $k \rightarrow \infty$. We want to prove that $P_{s_{k}} \rightarrow P \pi_{t_{0}}{ }^{-1}$. Fix, for some time, two positive numbers $\varepsilon$ and $\eta$. Since there are at most finitely many points $t$ for which

$$
P\{x:|x(t)-x(t-)| \geqq \varepsilon\} \geqq \eta
$$

holds, we can find an integer $k_{0}$ such that

$$
P A_{k}>1-\eta \quad \text { for all } k \geqq k_{0},
$$

where we have put

$$
A_{k}=\left\{x:\left|x\left(s_{k}\right)-x\left(s_{k}-\right)\right|<\varepsilon\right\} .
$$

Choose a compact set $K_{k}$ with $K_{k} \subset A_{k}$ and $P K_{k}>1-\eta$. To any function $x$ in $K_{k}$ we choose two positive numbers $\delta_{x}$ and $h_{x}$ such that the oscillation of any function from $S\left(x, \delta_{x}\right)$ over the interval $\left[s_{k}-h_{x}, s_{k}+h_{x}\right]$ is less than $\varepsilon$. Finitely many of the spheres, say $S\left(x_{1}, \delta_{x_{1}}\right), \ldots, S\left(x_{r}, \delta_{x_{r}}\right)$, cover $K_{k}$. Put 


$$
G_{k}=S\left(x_{1}, \delta_{x_{1}}\right) \cup \ldots \cup S\left(x_{r}, \delta_{x_{r}}\right) \quad \text { and } \quad h_{k}=\min \left\{h_{x_{1}}, \ldots, h_{x_{r}}\right\} \text {. }
$$

Then $G_{k}$ is open, $G_{k}$ contains $K_{k}$ and any function in $G_{k}$ oscillates by less than $\varepsilon$ in the interval $\left[s_{k}-h_{k}, s_{k}+h_{k}\right]$. Now choose a point $t_{k}$ in the interval $\left[s_{k}-h_{k}, s_{k}+h_{k}\right]$ such that $t_{k}>t_{0},\left|t_{k}-s_{k}\right|<1 / k$ and such that $t_{k} \in T_{P}$. Let $E$ be any Borel subset of $\mathrm{R}$. Then, for $k \geqq k_{0}$ the following inclusion holds:

$$
\left\{x: x\left(s_{k}\right) \in E\right\} \subset\left\{x: x\left(t_{k}\right) \in E^{\varepsilon}\right\} \cup G_{k}^{c} .
$$

Here $E^{\varepsilon}$ denotes the $\varepsilon$-neighbourhood of $E$, that is, the set of points within distance less than $\varepsilon$ from $E$, and ${ }^{c}$ indicates complementation. Considering a $k \geqq k_{0}$ and using the weak convergence $P_{n} \pi_{t_{k}}{ }^{-1} \rightarrow P \pi_{t_{k}}{ }^{-1}$ (which follows since $t_{k} \in T_{P}$ ), we now find that

$$
\begin{aligned}
P_{s_{k}}\left(\stackrel{\circ}{E)} \leqq \liminf _{n \rightarrow \infty} P_{n} \pi_{s_{k}}{ }^{-1}(E)\right. & \left.\leqq \limsup _{n \rightarrow \infty} P_{n} \pi_{t_{k}}{ }^{-1} \overline{\left(E^{\varepsilon}\right.}\right)+\limsup _{n \rightarrow \infty} P_{n}\left(G_{k}{ }^{c}\right) \\
& \left.\left.\leqq P \pi_{t_{k}}{ }^{-1} \overline{\left(E^{\varepsilon}\right.}\right)+P\left(G_{k}^{c}\right) \leqq P{\pi_{t_{k}}}^{-1} \overline{\left(E^{\varepsilon}\right.}\right)+\eta .
\end{aligned}
$$

Since $t_{k} \downarrow t_{0}$, the projections $\pi_{t_{k}}$ converge everywhere to $\pi_{t_{0}}$ as $k \rightarrow \infty$, hence

$$
P \pi_{t_{k}}{ }^{-1} \rightarrow P \pi_{t_{0}}{ }^{-1} \quad \text { as } k \rightarrow \infty .
$$

From the above inequality we thus find

$$
\begin{aligned}
\limsup _{k \rightarrow \infty} P_{s_{k}}(\stackrel{\circ}{E}) \leqq \limsup _{k \rightarrow \infty} P \pi_{t_{k}}{ }^{-1} \overline{\left(E^{\varepsilon}\right)}+\eta & \leqq P \pi_{t_{0}}{ }^{-1}\left(\overline{E^{\varepsilon}}\right)+\eta \\
& \leqq P \pi_{t_{0}}{ }^{-1}\left(E^{2 \varepsilon}\right)+\eta .
\end{aligned}
$$

Since this holds for all positive $\varepsilon$ and $\eta$, we finally find that

$$
\lim \sup P_{s_{k}}(\stackrel{\circ}{E}) \leqq P \pi_{t_{0}}{ }^{-1}(\bar{E}) \text {. }
$$

This being so for any $E$, we conclude by Theorem 1 that $P_{s_{k}} \rightarrow P_{\pi_{t_{0}}}{ }^{-1}$.

\section{Acknowledgement.}

I have had helpful discussions with K. R. Parthasarathy who suggested to me the two problems stated in the introduction; he was led to these problems by observing an error in the book by Gihman and Skorohod (p. 578 in [2]), where these authors seem to rely on a result like Theorem 2 without observing the difficulties involved.

\section{REFERENCES}

1. P. Billingsley, Weak convergence of probability measures, New York, 1968.

2. I. I. Gihman and A. V. Skorohod, Introduction to the theory of random processes, Izdat. "Nauka", Moscow, 1965. (Russian.) 
3. G. W. Mackey, Borel structure in groups and their duals, Trans. Amer. Math. Soc. 85 (1957), 134-165.

4. K. R. Parthasarathy, Probability measures on metric spaces, New York, 1967.

5. Yu. V. Prohorov, Convergence of random processes and limit theorems in probability, Theor. Probability Appl. 1 (1956), 157-214.

6. A. V. Skorohod, Limit theorems for stochastic processes, Theor. Probability Appl. 1 (1956), 261-290.

UNIVERSITY OF COPENHAGEN, DENMARK 